of looking straight, occasionally in elevation and depression, with no double vision in the terminal abduction right and left. The girl did not notice the pain. The pupil was in the mydriasis with a very slow motion in the light. Other status of the anterior eye segment as well as of the eye fundus was normal. A computed tomography scan showed a metallic foreign body in the left orbital conus, without fractures or intracranial intrusion. Immediate tetanus prophylaxis, antimicrobial therapy (amoxicillin/clavulanic acid, metronidazole), neuroprotective therapy (methylprednisolone) and local therapy (tobramycinum) were introduced. Given the high risk of surgical treatment, the girl was conservatively treated and monitored. The girl was relieved of physical activity at school due to the possibility of moving the foreign body. Three years after the injury, the girl has only a pupil in semi mydriasis and a slower reaction to direct light. The visual acuity is as follows: VOD sc 1.0, VOS sc 0.9. Other eye status is normal.

\section{SKIN BRIDGING AS A RESULT OF UNTREATED ONYCHOCRYPTOSIS IN A TEN-YEAR-OLD BOY}

Marko Bašković*, Ivan Petračić. Children's Hospital Zagreb

\subsection{6/archdischild-2021-europaediatrics.14}

A 10-year-old boy was examined for a problem with his right big toe that had been present for two years. In the family history there was no onychocryptosis. The child had not presented any worthy of note diseases in the past history. For two years he had presented a painful swelling of the lateral folds of the right big toe with repeated episodes of suppurative inflammation. The latter had always been treated with topical antiinflammatory and antibiotic therapy. He had never undergone surgery for fear of the scalpel. Physical examination showed a distally dystrophic nail with blood crusts at the level of the lateral grooves. The periungual skin presented chronic inflammation and above all formed cutaneous bridge that passed over the nail. The child underwent radical removal of the nail and its matrix under local anaesthesia.

The ingrown toenail of the big toe is a non-exceptional condition in children. When not treated properly it can become chronic. As in our case, the fear of the needle and the scalpel, which is frequent in children, can contribute to the chronicity. When chronic onychocryptosis is not treated adequately, it can be responsible for fibrous lesions, skin bridges and keloids. Although few cases of supraungual skin bridges have been described so far, it is known that hypertrophic granulation tissue can give rise to fibrous tissue covered by epidermis and then to skin bridges that join the two lateral folds. Two pathogenetic mechanisms have been hypothesized for this condition as follows: fusion of the highly inflamed lateral folds and penetration of the nail plate into the distal nail groove.

\section{QUALITATIVE ANALYSIS OF HOSPITAL MIDWIVES' CHALLENGES IN PROVIDING BREASTFEEDING-SUPPORT: IMPLICATIONS FOR IMPROVING POLICIES- IMPLEMENTATION PROCESS}

Jelena Dimnjaković*, Marija Švajda, Tamara Poljičanin. Croatian Institute of Public Health

10.1136/archdischild-2021-europaediatrics.15
Organization and policy declarations and documents support breastfeeding within Croatian healthcare from early pregnancy until long after birth.

However, results regarding the implementation efficiency and existing policy gaps are still missing. We aimed to identify hospital midwives' challenges in providing breastfeeding support and to recognize implementation policy gaps in hospitals in Croatia, in the field of breastfeeding.

Qualitative study designed in line with COREQ criteria, using semi-structured interviews in 12 healthcare professionals working in delivery room and maternity ward in 1 clinical hospital. Content was analyzed inductively by 3 researchers.

The analyses identified 5 themes: 1. self-awareness of hospital-midwives regarding their importance and success in breastfeeding-establishment, 2 . focusing on mother's motivation instead of on problem solving; 3. lack of continuity of care within the healthcare system, 4. lack of standard operating procedures and other organizational issues, 5. satisfaction of hospital staff with their level of knowledge despite lacking of breastfeeding education.

The hospital's midwives invest lots of resources and individual efforts in breastfeeding management, however, the interventions are not efficient, and the staff has no firm guidance on which topics to focus their efforts on.

Possible areas for intervention are day-to-day work organization, structuring in-house education, new employees' utilization, influencing midwifery-schools' curricula, and establishing standard operating procedures to help the staff to deal with the most common breastfeeding problems. National breastfeeding policy does not seem to be fully and efficiently implemented in Croatia. In order to tackle breastfeeding challenges, revision of existing policies, good implementation management and clear definition of responsibilities regarding breastfeeding protection and promotion in hospitals are needed.

\section{6 COMPARISON OF CHILDREN WITH KAWASAKI DISEASE AND MIS-C}

Laura Prtorić*, Diana Didović, Ivana Valenčak-Ignjatić, Ante Šokota, Lorna StembergerMarić. University Hospital for Infectious Diseases "Dr. Fran Mihaljević"

\subsection{6/archdischild-2021-europaediatrics. 16}

The goal of this research was to compare clinical signs, symptoms and laboratory findings in children diagnosed with Kawasaki disease and those with multisystem inflammatory syndrome in children (MIS-C) associated with coronavirus disease 2019 (COVID-19).

We reviewed medical records of children diagnosed with Kawasaki disease or MIS-C hospitalised in the University Hospital for Infectious Diseases "Dr. Fran Mihaljević" in the period from February 25th 2020 to February 24th 2021. We defined MIS-C using World Health Organisation criteria.

There were 13 children diagnosed with Kawasaki disease and 23 children diagnosed with MIS-C hospitalised in this period. The average duration of hospitalisation was similar in both groups, approximately 8 days. Boys were overall more affected $(66 \%)$ than girls. Children with MIS-C were older and more often gastrointestinal symptoms were present. When comparing laboratory results, children in the MIS-C group had higher C-reactive protein levels and lower platelet count. Also, they required intensive care treatment more frequently. The first therapy of choice for all children with Kawasaki 